12,13

\title{
Адсорбция атомов кремния на поверхность системы Au/W(110)
}

\author{
(ㄱ Д.А. Пудиков, Е.В. Жижин, А.А. Вишнякова, О.Ю. Вилков, Г.Г. Владимиров
}

Санкт-Петербургский государственный университет,

Санкт-Петербург, Россия

E-mail: gelbry@mail.ru

(Поступила в Редакцию 27 марта 2017 г.)

\begin{abstract}
Методом фотоэлектронной спектроскопии с угловым разрешением и рентгеновской фотоэлектронной спектроскопией исследована возможность формирования упорядоченной структуры, схожей с силиценом, на поверхности $\mathrm{Au} / \mathrm{W}(110)$. Показано, что добавление атомов кремния приводит к заметному искажению электронной структуры исходной подложки и сформированная электронная структура не соотносится с силиценом. При этом конфигурация рефлексов на картине дифракции медленных электронов свидетельствует об образовании упорядоченных двумерных кремниевых структур с большим числом разнонаправленных доменов.
\end{abstract}

Исследования проводились на оборудовании Ресурсного центра „Физические методы исследования поверхности“ Научного парка СПбГУ.

DOI: 10.21883/FTT.2017.12.45246.092

\section{1. Введение}

Тенденции развития современной наноэлектроники по-прежнему определяют устройства на основе кремния. На протяжении нескольких десятилетий основная из них была направлена на масштабирование полупроводниковых устройств с повышением рабочих характеристик и снижением энергопотребления микросхем. При таком подходе не теряет свою актуальность разработка и создание новых наноструктур. Наиболее перспективным материалом, призванным заменить кремний в микросхемах, считается графен вследствие его выдающихся физикохимических свойств, которые обусловлены конической электронной структурой в окрестности точки Дирака на уровне Ферми [1-4]. Одним из ключевых препятствий на пути массового внедрения графена в наноэлектронику является отсутствие высокоэффективных и экономически выгодных методов синтеза при низких температуpax $[5,6]$, а также ограниченность в выборе подложек. В связи с этим одним из приоритетных направлений является нахождение нового графеноподобного двумерного материала для более широкого класса подложек. Кремниевым подобием графена считается силицен $[7,8]$. Расчеты на основе теории функционала плотности показали, что атомы кремния могут формировать упорядоченную двумерную гексагональную корругированную структуру $[9,10]$, которая, так же как и графен, может иметь широкий спектр применений в наноэлектронике. Например, полевой транзистор [11], электрод для литийионных аккумуляторов [7]. Кроме того, силицен может быть синтезирован и преобразован с использованием базовых технологий полупроводниковой индустрии, тем самым упрощая процесс интеграции в существующую электронику.

В работах по эпитаксиальному росту кремния на подложках $\mathrm{ZrB}_{2}$ [12], $\mathrm{Ag}(110)$ [13], $\operatorname{Ir}(111)$ [14], $\mathrm{Pt}(111)$ [15] были исследованы упорядоченные структуры кремния, схожие с силиценом. И лишь недавно в работе [16] была наглядно продемонстрирована электронная и кристаллическая структура силицена на поверхности $\mathrm{Ag}(111)$.

Настоящая работа посвящена изучению возможности формирования упорядоченной структуры, схожей с силиценом, на поверхности $\mathrm{Au} / \mathrm{W}(110)$. С одной стороны, в работе [17] показана возможность низкотемпературной миграции кремния на поверхности $\mathrm{W}(100)$ с образованием поверхностного силицида вольфрама WSi. С другой стороны, система $\mathrm{Au} / \mathrm{W}(110)$ является хорошо упорядоченной и исследованной [18-20]. Стоит ожидать, что взаимодействие монослоя (ML) золота и вольфрама уменьшит вероятность образования сплава $\mathrm{Si}-\mathrm{Au}$ и в то же самое время будет обеспечиваться условие для миграции атомов кремния на поверхности золота за счет остаточного влияния подложки вольфрама, приводя к образованию упорядоченной структуры на основе кремния.

\section{2. Экспериментальные детали}

Исследования проводились на аналитическом модуле фотоэлектронной спектроскопии научно-исследовательской платформы Нанолаб Ресурсного центра „Физические методы исследования поверхности“ Научного парка Санкт-Петербургского государственного университета.

Электронная структура измерялась методом фотоэлектронной спектроскопии с угловым разрешением (ФЭСУР) и рентгеновской фотоэлектронной спектроскопией (РФЭС). Информация о кристаллической структуре была получена с помощью дифракции медленных электронов (ДМЭ). Измерения проводились при комнатной температуре. Базовое давление в камере во время эксперимента не превышало $2 \cdot 10^{-10}$ mbar. 
Атомарно-чистая поверхность монокристалла W(110) была получена за счет проведения серии чередующихся прогревов в атмосфере кислорода (парциальное давление $\left.\mathrm{O}_{2} \sim 5 \cdot 10^{-8} \mathrm{mbar}, 1250^{\circ} \mathrm{C}, 20 \mathrm{~min}\right)$ и кратковременных высокотемпературных отжигов $\left(1650-1800^{\circ} \mathrm{C}\right.$, $20 \mathrm{sec})$ в сверхвысоком вакууме [17-19]. На подготовленную подложку $\mathrm{W}(110)$ осаждался $1 \mathrm{ML}$ золота $(2.3 \AA)$ с последующим прогревом $\left(300^{\circ} \mathrm{C}, 20 \mathrm{~min}\right)$. На сформированную систему $\mathrm{Au} / \mathrm{W}(110)$ осаждался слой кремния ( $2 \mathrm{ML}$ или $2.7 \AA)$ с последующим прогревом $\left(350^{\circ} \mathrm{C}\right.$, $20 \mathrm{~min}$ ) для формирования упорядоченной структуры. Напыления проводились при комнатной температуре.

Скорость напыления оценивалась по кварцевым микровесам и составляла $\sim 1 \AA / \min$. Помимо скорости напыления толщина слоя золота контролировалась за счет формирования характерного для $1 \mathrm{ML}$ квантового электронного состояния (КЭС) в валентной зоне [18-21].

Для записи дисперсионных зависимостей в качестве источника излучения использовалась ультрафиолетовая газоразрядная лампа с монохроматором (He II, энергия фотонов $40.8 \mathrm{eV})$.

\section{3. Результаты и обсуждение}

Для характеризации кристаллической структуры до и после адсорбции атомов кремния были получены картины ДМЭ (рис. 1). Для исходной поверхности $\mathrm{Au} / \mathrm{W}(110)$ (рис. 1,a) на картине дифракции видны шесть четких рефлексов, расположенных в форме правильного шестиугольника вокруг основного рефлекса. Такая картина соотносится с реконструкцией поверхности $p(9 \times 9)$ и соответствует однодоменному золоту большой площади, осажденному на подложку вольфрама, и не содержит дополнительных рефлексов от различных загрязнений.

На рис. 1,b представлена картина дифракции после адсорбции атомов кремния на $\mathrm{Au} / \mathrm{W}(110)$ и последующего прогрева. Поверхность системы $\mathrm{Si} / \mathrm{Au} / \mathrm{W}(110)$ по-прежнему хорошо упорядочена, о чем свидетельствуют четкие основные рефлексы гексагона. При этом на поверхности формируется упорядоченная кристалличе-
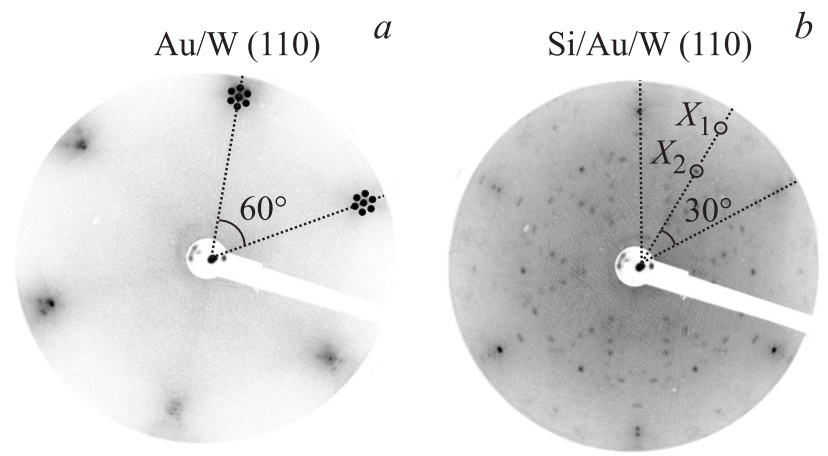

Рис. 1. Картины ДМЭ для систем: $(a)-\mathrm{Au} / \mathrm{W}(110)$ при $E_{p}=65 \mathrm{eV}$ и $(b)-\mathrm{Si} / \mathrm{Au} / \mathrm{W}(110)$ при $E_{p}=65 \mathrm{eV}$.

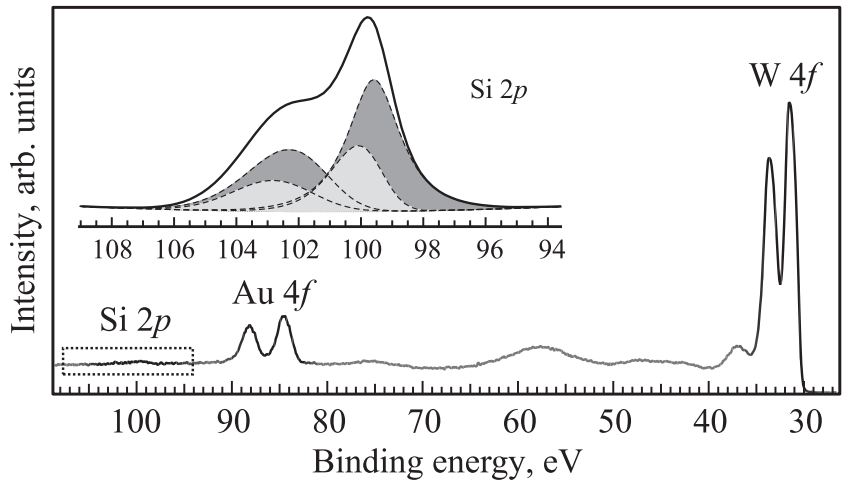

Рис. 2. РФЭС спектры для системы $\mathrm{Si} / \mathrm{Au} / \mathrm{W}(110)$ : обзорный и линия $\mathrm{Si} 2 \mathrm{p}$ (на вставке).

ская структура с элементами симметрии шестого порядка. Особое внимание в этой структуре привлекают слабовыраженные рефлексы ( $x_{1}$ и $\left.x_{2}\right)$, повернутые на $30^{\circ}$ относительно четких рефлексов. Кроме того, между рефлексами $x_{2}$ можно выделить слабовыраженные сдвоенные рефлексы. Подобная конфигурация рефлексов свидетельствует об образовании упорядоченных двумерных кремниевых структур на поверхности с большим числом разнонаправленных доменов, подобно реконструкциям $\left((4 \times 4),(\sqrt{13} \times \sqrt{13}) R 13.9^{\circ},(2 \sqrt{3} \times 2 \sqrt{3}) R 30^{\circ}\right)$ силицена на поверхности $\operatorname{Ag}(111)$ [16].

На рис. 2 представлен обзорный спектр РФЭС и линия $\mathrm{Si} 2 \mathrm{p}$ для системы $\mathrm{Si} / \mathrm{Au} / \mathrm{W}(110)$. В структуре линии $\mathrm{Si} 2 \mathrm{p}$ можно выделить два спин-орбитальных дублета. Основной вклад в структуру линии вносит дублет с энергией связи $99.6 \mathrm{eV}$, другой расположен при $102.3 \mathrm{eV}$. Количественная оценка толщины кремниевой пленки на поверхности системы $\mathrm{Au} / \mathrm{W}(110)$ дает значение $\sim 2.4 \mathrm{ML}$ или $0.38 \mathrm{~nm}$.

Дисперсии для трех высокосимметричных направлений $(\boldsymbol{\Gamma S}, \boldsymbol{\Gamma N}, \boldsymbol{\Gamma H})$ поверхностной зоны Брюллиэна (ПЗБ) для системы (1 ML)-Au/W(110) показаны на рис. 3. В области 0-1 eV формируются интерфейсные состояния, находящиеся в запрещенной зоне вольфрама $\left(k_{\|}>0.3 \AA^{-1}\right)$. Формирование интерфейсных состояний характерно при покрытии монокристалла W(110) $1 \mathrm{ML}$ благородного металла [18,21]. Данные состояния свидетельствуют о высокой степени кристаллической упорядоченности и отсутствии поверхностных загрязнений подложки и напыляемого металла, а также об определенной толщине напыленной пленки. В электронной структуре также отчетливо проявляются $d$-состояния вольфрама, имеющие в точке Г энергии связи 0.5 и $1 \mathrm{eV}$ и увеличивающиеся по энергии с возрастанием $k_{\|}$.

На рис. 4 представлены дисперсии электронных состояний системы $\mathrm{Au} / \mathrm{W}(110)$ (слева) и $\mathrm{Si} / \mathrm{Au} / \mathrm{W}(110)$ (справа) в направлении ГN ПЗБ. Для системы (1 ML)-Au/W(110) характерно наличие KЭC $d$-характера, имеющих в точке Г энергии связи 3.1 и $3.7 \mathrm{eV}$, а также КЭС $s, p$-характера, расположенных при $4.9 \mathrm{eV}$ [21]. После напыления $1 \mathrm{ML}$ кремния картина дисперсии 

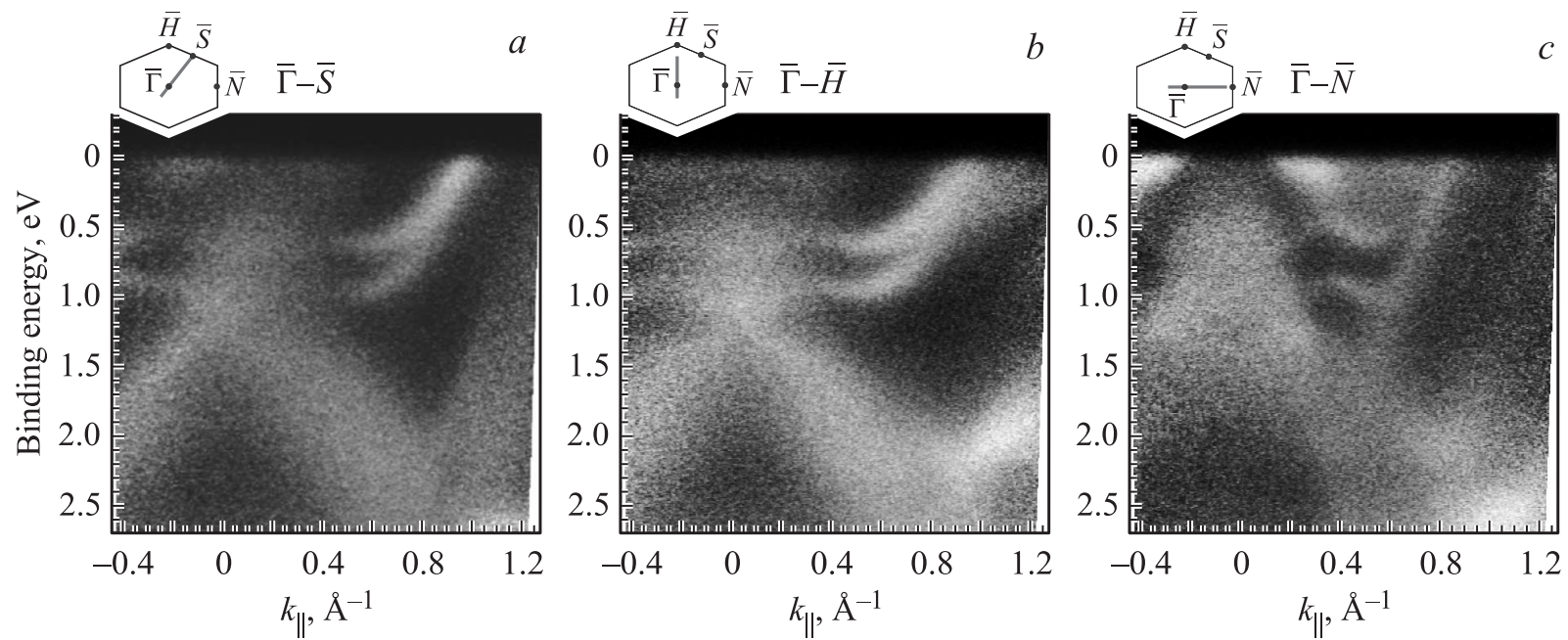

Рис. 3. Электронная структура (1 ML) Au/W(110) в направлениях $\mathbf{\Gamma S}-(a), \mathbf{\Gamma N}-(b), \mathbf{\Gamma H}-(c)$ ПЗБ.
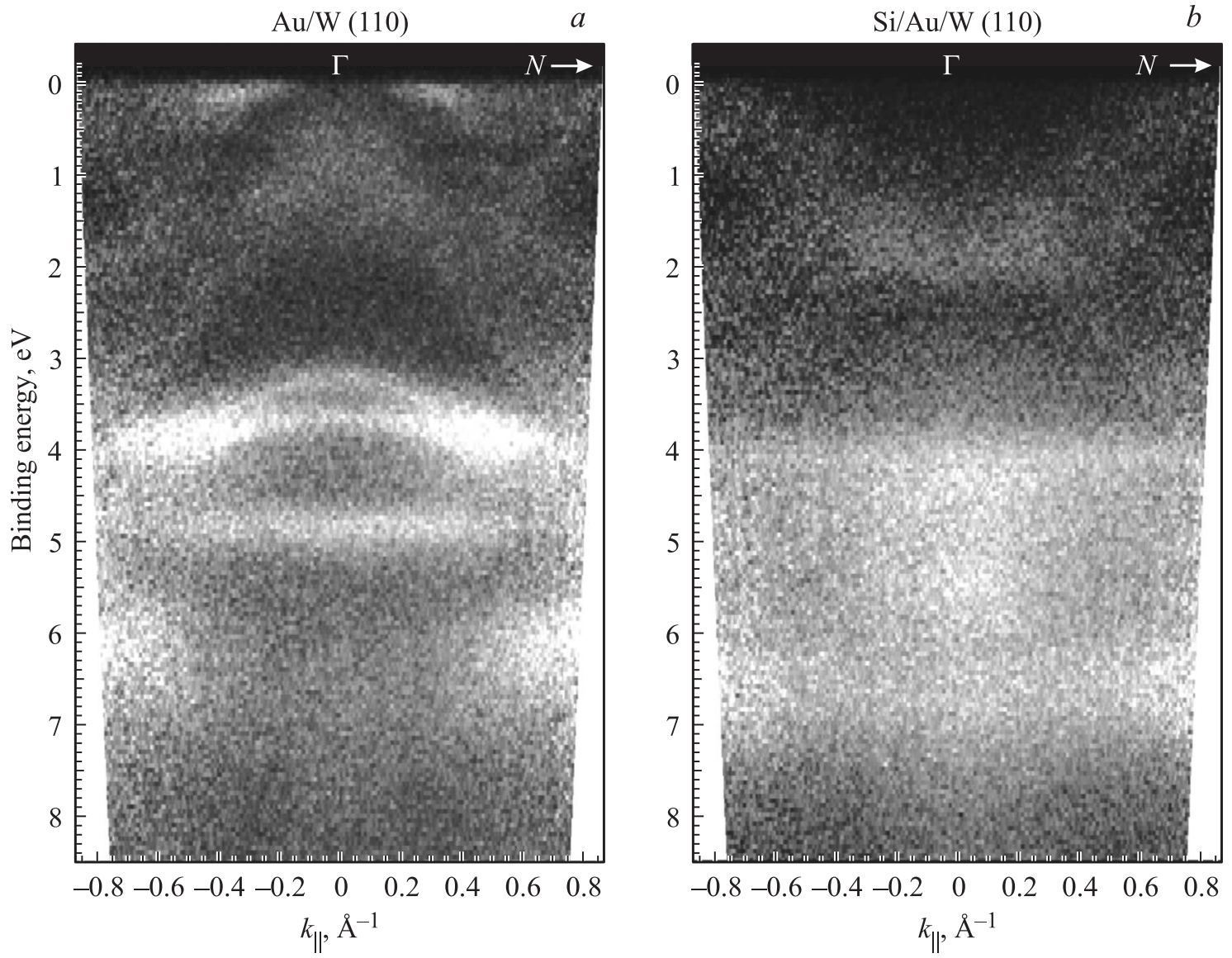

Pис. 4. Электронная структура для систем: $\mathrm{Au} / \mathrm{W}(110)(a)$ и $\mathrm{Si} / \mathrm{Au} / \mathrm{W}(110)-(b)$ в направлении ГN ПЗБ.

электронных состояний сильно изменяется. Заметно уменьшается интенсивность состояний вольфрама в области $0.5-1.5 \mathrm{eV}$ и электронных состояний интерфейса $\mathrm{Au}-\mathrm{W}$ в области 0-1.5 eV. Полностью изменяется вид КЭС с энергиями $3.0-5.0 \mathrm{eV}$ : в системе $\mathrm{Si} / \mathrm{Au} / \mathrm{W}(110)$ формируются плоские зоны с энергией связи 4.1 и $6.8 \mathrm{eV}$.
Электронная структура системы в направлении ГН ПЗБ до и после напыления кремния представлена на рис. 5. Система $\mathrm{Au} / \mathrm{W}(110)$ имеет характерную дисперсию электронных состояний: видны $d$-состояния вольфрама и интерфейсные состояния данной системы, о чем уже было сказано выше. 

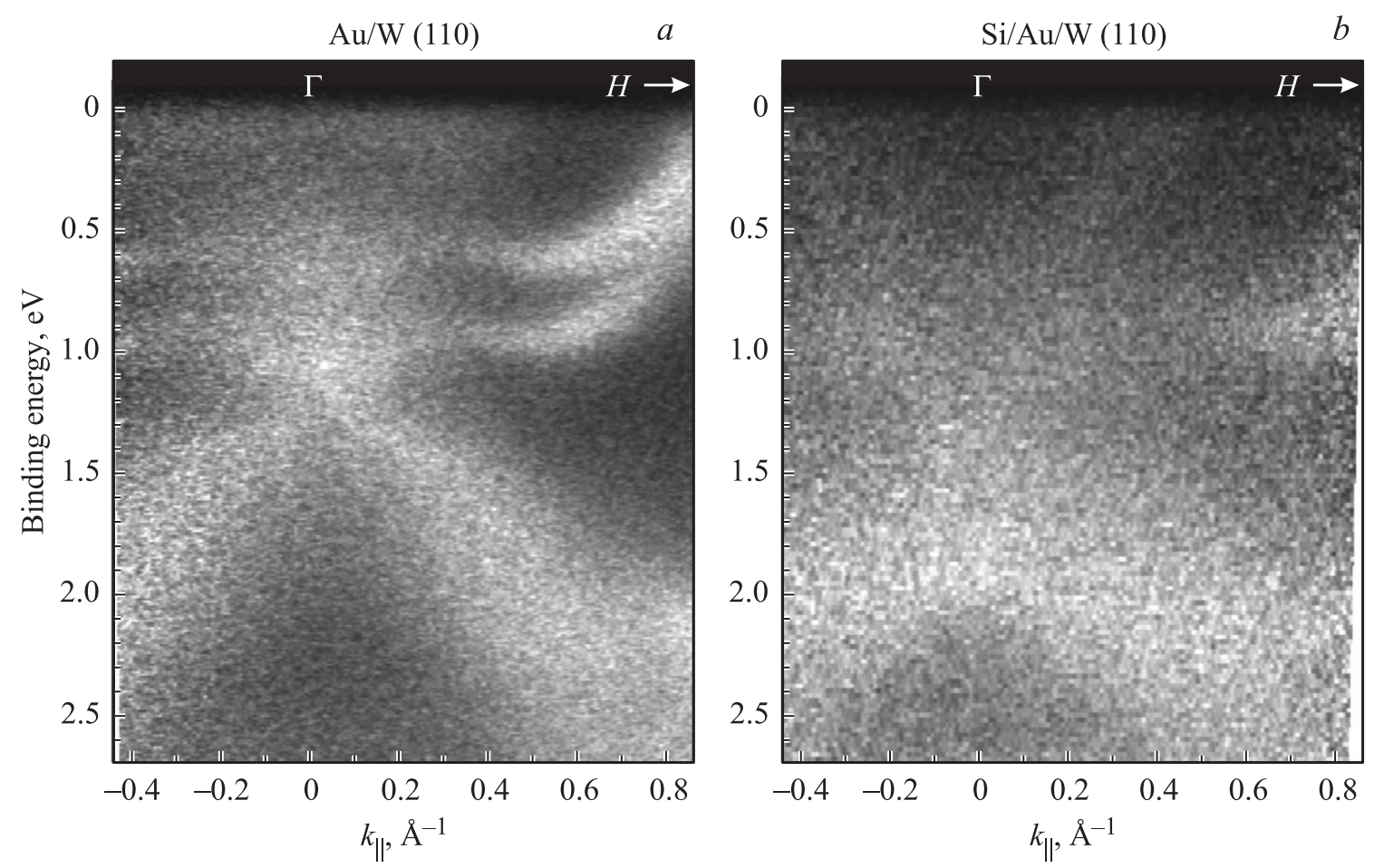

Рис. 5. Электронная структура для систем: $\mathrm{Au} / \mathrm{W}(110)-(a)$ и $\mathrm{Si} / \mathrm{Au} / \mathrm{W}(110)-(b)$ в направлении ГН ПЗБ.

Адсорбция атомов кремния приводит к формированию общего состояния с $5 d$-состоянием золота с энергией $\sim 1.0 \mathrm{eV}$, которое также локализовано в области запрещенной зоны исходной вольфрамовой подложки, т.е. при $k_{\|}>0.3 \AA^{-1}$. В области точки $\Gamma$ электронная структура схожа с электронной структурой вольфрама [20]. Прослеживается параболическое состояние с вершиной в точке $\boldsymbol{\Gamma}$ при энергии $0.7 \mathrm{eV}$, ветви которого пересекают уровень Ферми на $k_{\|} \sim 0.4 \AA^{-1}$. При этом наблюдается формирование плоской зоны с энергией связи $\sim 2.1 \mathrm{eV}$.

Таким образом, добавление атомов кремния приводит к смешиванию электронных состояний с золотом в валентной зоне без формирования уникальных особенностей электронных состояний, в том числе характерных для силицена $[8,16]$.

\section{4. Заключение}

Методами УФЭС и РФЭС исследована возможность формирования упорядоченной структуры, схожей с силиценом, на поверхности $\mathrm{Au} / \mathrm{W}(110)$. Показано, что электронная структура исходной поверхности $\mathrm{Au} / \mathrm{W}(110)$ хорошо согласуется с результатами многих работ. Адсорбция атомов кремния приводит к заметному искажению электронной структуры исходной подложки. После напыления атомов кремния исчезают интерфейсные состояния в запрещенной зоне вольфрама, а вместо них формируется общее состояние с энергией $\sim 1.0 \mathrm{eV}$, локализованное в области запрещенной зоны исходной вольфрамовой подложки. В электронной структуре так- же формируются плоские зоны. Таким образом, добавление атомов кремния приводит к обычному смешиванию электронных состояний с золотом в валентной зоне без формирования уникальных особенностей электронных состояний, в том числе характерных для силицена.

В то же самое время картина ДМЭ свидетельствует об образовании упорядоченных двумерных кремниевых структур на поверхности $\mathrm{Au} / \mathrm{W}(110)$ с большим числом разнонаправленных доменов.

\section{Список литературы}

[1] A.H. Castro Neto, F. Guinea, N.M.R. Peres, K.S. Novoselov, A.K. Geim. Rev. Mod. Phys. 81, 109 (2009).

[2] M. Batzill. Surf. Sci. Rep. 67(3-4), 83 (2012).

[3] O.V. Yazyev. Rep. Prog. Phys. 73, 056501 (2010).

[4] E.V. Zhizhin, A. Varykhalov, A.G. Rybkin, A.A. Rybkina, D.A. Pudikov, D. Marchenko, J. Sánchez-Barriga, I.I. Klimovskikh, G.G. Vladimirov, O. Rader, A.M. Shikin. Carbon 93, 984 (2015).

[5] E.V. Zhizhin, D.A. Pudikov, A.G. Rybkin, A.E. Petukhov, Y.M. Zhukov, A.M. Shikin. Mater. Des. 104, 284 (2016).

[6] Е.В. Жижин, Д.А. Пудиков, А.Г. Рыбкин, П.Г. Ульянов, А.М. Шикин. ФТТ 57, (9), 1839 (2015).

[7] H. Oughaddou, H. Enriquez, M.R. Tchalala, H. Yildirim, A.J. Mayne, A. Bendounan, G. Dujardin, M.A. Ali, A. Kara. Prog. Surf. Sci. 90, 46 (2015).

[8] A. Kara, H. Enriquez, A.P. Seitsonend, L.C. Lew Yan Voone, S. Vizzini, B. Aufrayg, H. Oughaddoub. Surf. Sci. Rep. 67, 1 (2012).

[9] K. Takeda, K. Shiraishi. Phys. Rev. B 50, 14916 (1994). 
[10] S. Cahangirov, M. Topsakal, E. Aktürk, H. Sahin, S. Ciraci. Phys. Rev. Lett. 102, 236804 (2009).

[11] L. Tao, E. Cinquanta, D. Chiappe, C. Grazianetti, M. Fanciulli, M. Dubey, A. Molle, D. Akinwande. Nature Nanotechnol. 10, 227 (2015).

[12] A. Fleurence, R. Friedlein, T. Ozaki, H. Kawai, Y. Wang, Y. Yamada-Takamura. Phys. Rev. Lett. 108, 245501 (2012).

[13] B. Aufray, A. Kara, S. Vizzini, H. Oughaddou, C. Léandri, B. Ealet, G. L. Lay. Appl. Phys. Lett. 96, 183102 (2016).

[14] L. Meng, Y. Wang, L. Zhang, S. Du, R. Wu, L. Li, Y. Zhang, G. Li, H. Zhou, W.A. Hofer, H. Gao. Nano Lett. 13 (2), 685 (2013).

[15] M.Švec, P. Hapala, M. Ondráček, P. Merino, M. Blanco-Rey, P. Mutombo, M. Vondraček, Y. Polyak, V. Cháb, J.A. Martìn Gago, P. Jelìnek. Phys. Rev. B 89, 201412(R) (2014).

[16] P.M. Sheverdyaeva, S.K. Mahatha, P. Moras, L. Petaccia, G. Fratesi, G. Onida, C. Carbone. ACS Nano 11, 975 (2017).

[17] В.Н. Агеев, Е.Ю. Афанасьева, Н.Р. Галль, С.Н. Михайлов, Е.В. Рутьков, А.Я. Тонтегоде. Поверхность 5, 7 (1987).

[18] A.M. Shikin, A. Varykhalov, G.V. Prudnikova, D. Usachov, V.K. Adamchuk, Y. Yamada, J.D. Riley, O. Rader. Phys. Rev. Lett. 100, 057601 (2008).

[19] A.M. Shikin, O. Rader, G.V. Prudnikova, V.K. Adamchuk, W. Gudat. Phys. Rev. B 65, 075403 (2002).

[20] A.M. Shikin, A.A. Rybkina, M.V. Rusinova, I.I. Klimovskikh, A.G. Rybkin, E.V. Zhizhin, E.V. Chulkov, E.E. Krasovskii. New J. Phys. 15, 125014 (2013).

[21] A.M. Shikin, A.A. Rybkina, A.S. Korshunov, Yu.B. Kudasov, N.V. Frolova, A.G. Rybkin, D. Marchenko, J. Sánchez-Barriga, A. Varykhalov, O. Rader. New J. Phys. 15, 095005 (2013). 